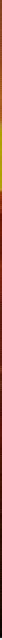

\title{
Aplicaciones en predicción aeronáutica
}

\section{1}

DOI: 10.31978/014-18-009-X.31

\author{
ERNEST WERNER HIDALGO \\ D. T. en Cataluña, Agencia Estatal de Meteorología (AEMET)
}

Todo despegue es opcional. Todos los aterrizajes son obligatorios.

Frase común en el mundo de la aviación - ANÓNIMO

La información meteorológica es esencial para la planificación y seguridad de las operaciones aéreas, tanto en los aeropuertos como en las rutas aéreas. Para minimizar los riesgos asociados a las condiciones atmosféricas se optimiza y reorganiza el tráfico y, a menudo, estas optimizaciones provocan una disminución de frecuencia de salidas y entradas de aviones, con retrasos y pérdidas económicas importantes. Para ayudar a la predicción de los parámetros de mayor impacto en la aviación y con la resolución horizontal cada vez más alta de los sistemas de predicción por conjuntos se han diseñado herramientas específicas para predicción aeronáutica, que presentamos en este capítulo.

Palabras clave: sistemas de predicción por conjuntos aplicados a la meteorología aeronáutica, EPSgramas ad hoc para pronósticos de aeródromo, EPSgramas aeronáuticos.

\footnotetext{
Imagen parte superior: aeropuerto de Jerez de la Frontera, el 17 octubre 2015. La imagen se tomó en dirección suroeste desde la ubicación del heliógrafo (situado en una intersección de la carretera perimetral interior próxima a la pista). Se observan cumulus y stratocumulus, con cielo algo caótico posterior al paso de un frente. Fueron las primeras lluvias de ese año hidrológico que comenzó en septiembre de 2015. Se pueden observar diferentes aeronaves emplazadas en un parking auxiliar del aeropuerto. Fotografía tomada por PEPE TORRES, con un Samsung Galaxy SII y posterior aplicación de un filtro de instagram.
} 


\subsection{Introducción}

La información meteorológica es esencial para la planificación y seguridad de las operaciones aéreas. Parámetros como la visibilidad, la nubosidad, el viento, la temperatura y el tiempo presente influyen significativamente en las operaciones diarias y determinan, por ejemplo, la configuración de las pistas en servicio, la longitud requerida para el despegue o el peso máximo permitido.

Uno de los parámetros que más influyen en las maniobras de despegue y aterrizaje de los aviones es el viento. En particular el viento en cola y el viento cruzado en pista, suponen un riesgo para estas maniobras. Por ejemplo, el viento en cola puede producir una pérdida de sostenibilidad del avión o un planeo mayor en el aterrizaje y el viento cruzado puede suponer una desestabilización de las aeronaves, sobre todo cuando es racheado.

Para evitar o minimizar estos riegos los encargados de la planificación de las operaciones aéreas cambian las cabeceras habituales de entrada y salida de los aviones. Este cambio implica una reorganización y una disminución de la frecuencia de las salidas y entradas de aviones con los consiguientes retrasos.

Con la finalidad de ayudar a la predicción de los parámetros que más afectan a la aviación se han diseñado los EPSgramas aeronáuticos que describimos a continuación.

\subsection{EPSgramas aeronáuticos}

Los EPSgramas aeronáuticos (se describen los EPSgramas en general en la sec. 27.8.1 en la página 425) son un ejemplo de aplicación práctica de los EPSgramas. Se trataría de dar información probabilista objetiva a un usuario aeronáutico ya sea un predictor, controlador aéreo o piloto. El usuario final dispondría de EPSgramas de parámetros generales, como por ejemplo la precipitación, la dirección y velocidad del viento o la humedad y también de otros parámetros estrictamente aeronáuticos, como podrían ser el viento en cola en una cabecera o la probabilidad de viento cruzado en una pista.

\subsection{EPSgramas con el ECENS}

Los primeros EPSgramas aeronáuticos se realizaron utilizando el sistema(s) de predicción por conjuntos (SPC) del European Centre for Medium-range Weather Forecasts -Centro Europeo de Predicción a Plazo Medio- (ECMWF), el ECENS (sec. 19.3 en la página 293). Este SPC tiene actualmente (2018) una resolución espacial de 18 kilómetros y temporal de 6 horas. Como punto de grid representativo del aeropuerto se escogió el punto más cercano con la única restricción de que estuviese situado en tierra. Es evidente que ni la resolución espacial ni la temporal son suficientemente buenas para cubrir las necesidades de un usuario aeronáutico, pero nos permiten ver la potencia de los EPSgramas para suministrar información probabilista gráficamente de forma compacta.

Se realizaron los siguientes tipos de gráficos:

- Para los parámetros generales como la Precipitación acumulada, Temperatura, Punto de rocío, $\mathrm{Nu}$ bosidad, Viento medio y Racha (Figura 31.1 en la página siguiente) se utilizó el formato boxplot o EPSgrama de cajas con los percentiles: 10, 25, 50 (mediana) 75 y 90 y los whiskers para los valores extremos.

- Para la Dirección de viento (Figura 31.2 en la página siguiente) se utilizó una distribución de las probabilidades de cada dirección por sectores de 45 grados con el área del sector proporcional a la probabilidad.

- Finalmente, para los EPSgramas aeronáuticos Viento en cola en una cabecera y Viento cruzado en una pista (Figura 31.3 en la página siguiente), se utilizaron diagramas de barras con un código de tres colores que dividen la probabilidad de viento según su intensidad: amarillo superior a tres nudos, naranja superior a 8 nudos y rojo superior a 16 nudos. En el caso del viento en cola se suman las proyecciones del viento en la dirección de la cabecera, mientras que en el caso del viento cruzado se representa la probabilidad del sector perpendicular a la pista. 


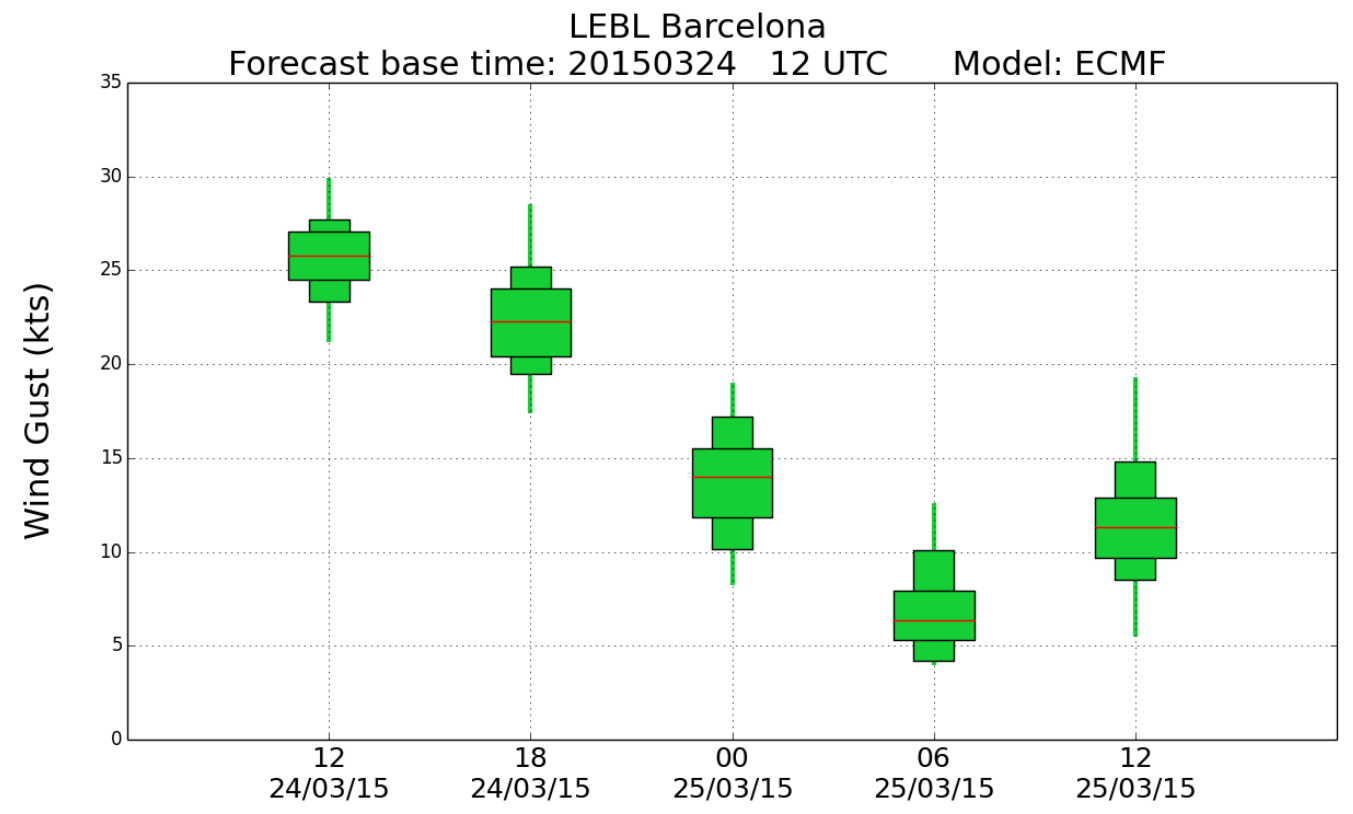

Figura 31.1: EPSgrama Racha de viento para LEBL (boxplot).

\section{LEBL Barcelona \\ Forecast base time: 2015032412 UTC Model: ECMF}

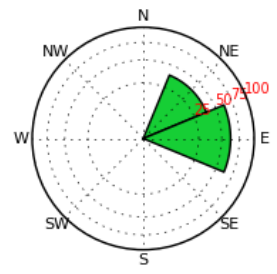

12

$24 / 03 / 15$

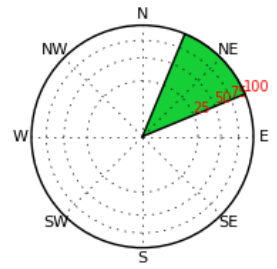

18

$24 / 03 / 15$

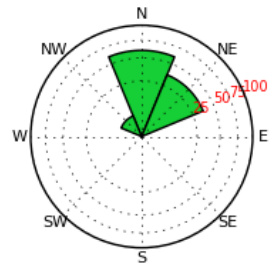

00

$25 / 03 / 15$

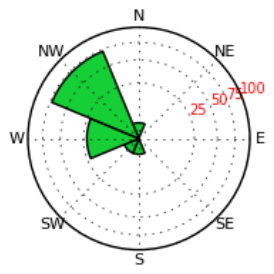

06

$25 / 03 / 15$

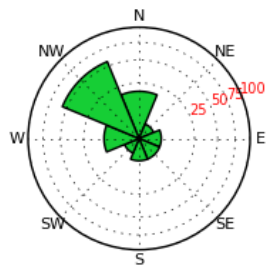

12

$25 / 03 / 15$

Wind direction

Figura 31.2: EPSgrama Dirección de viento para LEBL (probabilidad por sectores).
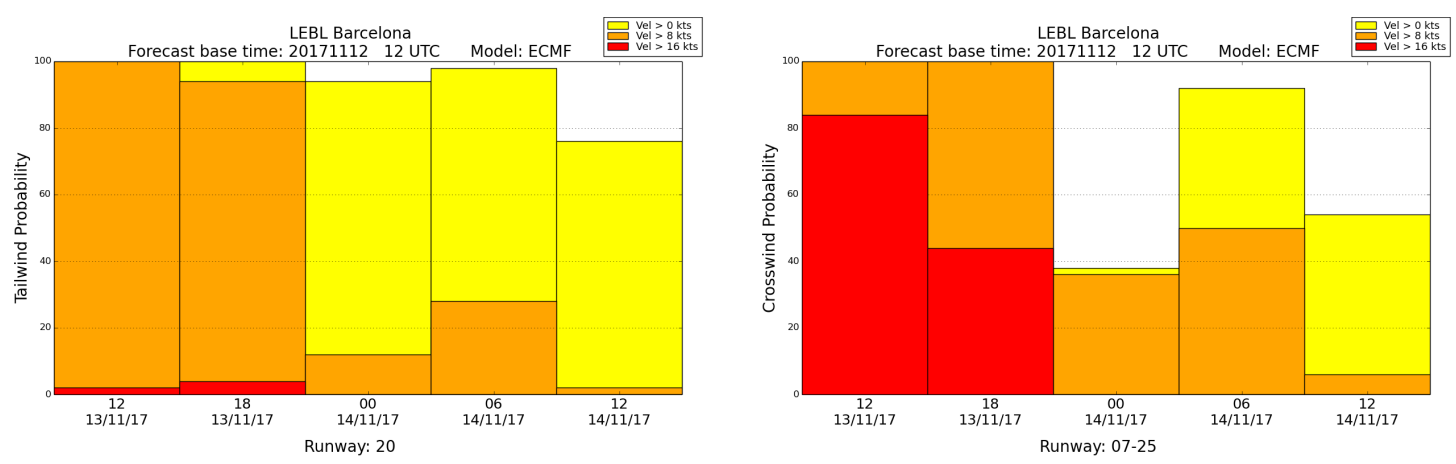

Figura 31.3: Izquierda: EPSgrama Viento en cola Cabecera 20 para LEBL (diagrama de barras). Derecha: EPSgrama Viento cruzado pista 07-25 para LEBL (diagrama de barras). 


\subsection{EPSgramas con GLAMEPS}

En el siguiente paso se utilizó el SPC GLAMEPS (sec. 20.2 en la página 304) que tiene una resolución temporal de 3 horas y espacial de $8 \mathrm{~km}$. Igualmente se escogió para la elaboración del EPSgrama el punto más cercano al aeropuerto que no cayese sobre el mar.

Ejemplos de los EPSgramas de parámetros generales basados en GLAMEPS se muestran en las Figuras 31.4 (nubosidad), 31.5 (temperatura) y 31.6 (pre- cipitación),

Por otro lado, ejemplos de los EPSgramas aeronáuticos con GLAMEPS pueden verse en las Figuras 31.7 (viento en cola) y 31.8 en la página siguiente (viento cruzado). Para el viento cruzado se introdujo un diagrama de barras doble donde el eje de las abscisas representa la dirección de la pista y la probabilidad de viento cruzado a cada lado viene dada por la altura de la barra. Los colores dan información de la velocidad del viento con la misma escala que el viento en cola.

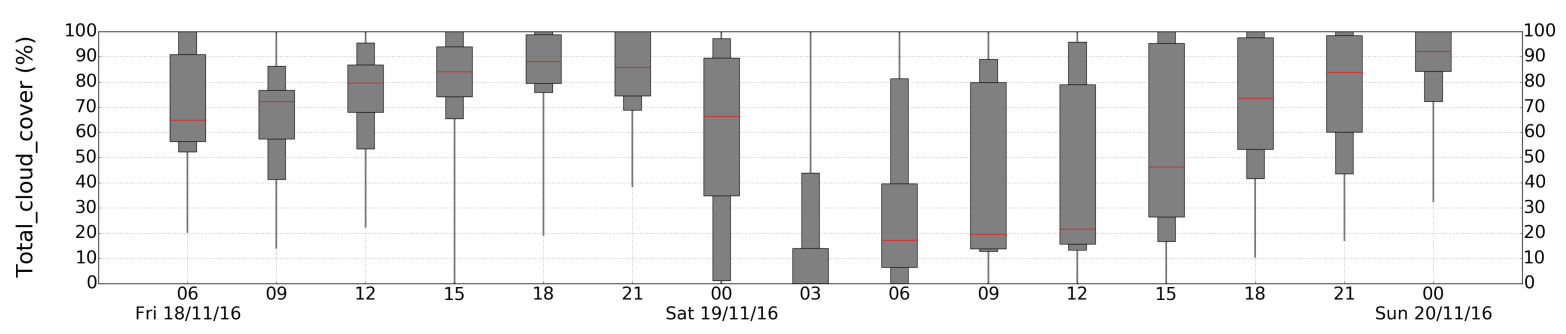

Figura 31.4: GLAMEPS: Nubosidad LEMD (boxplot).

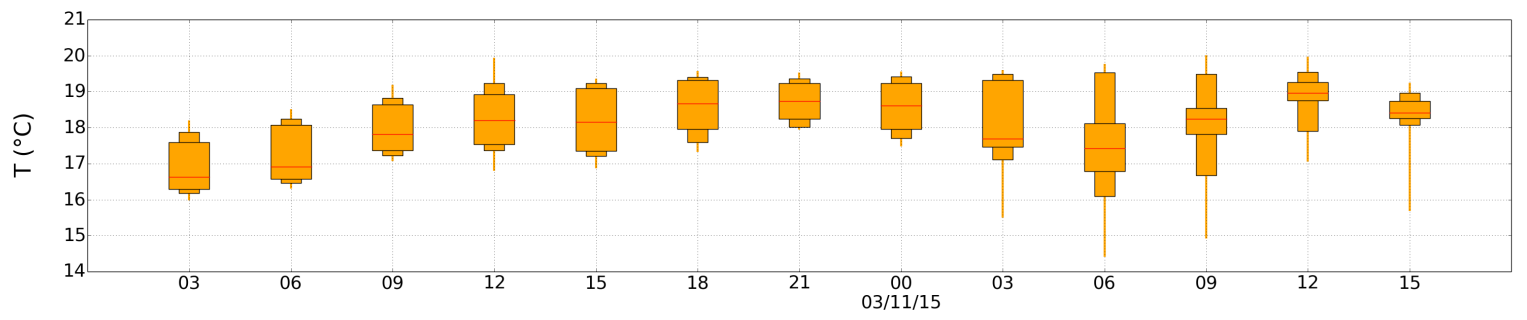

Figura 31.5: GLAMEPS: Temperatura LEBL (boxplot).

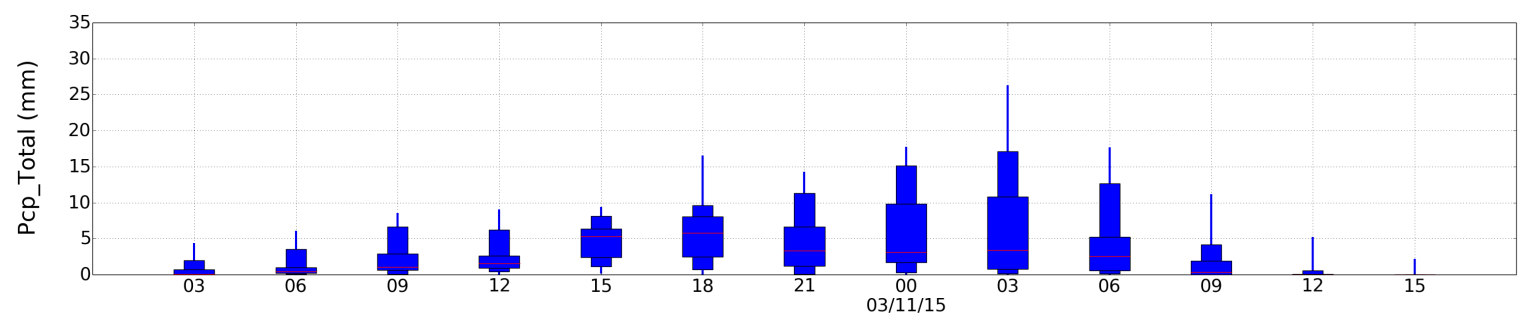

Figura 31.6: GLAMEPS: Precipitación LEBL (boxplot).

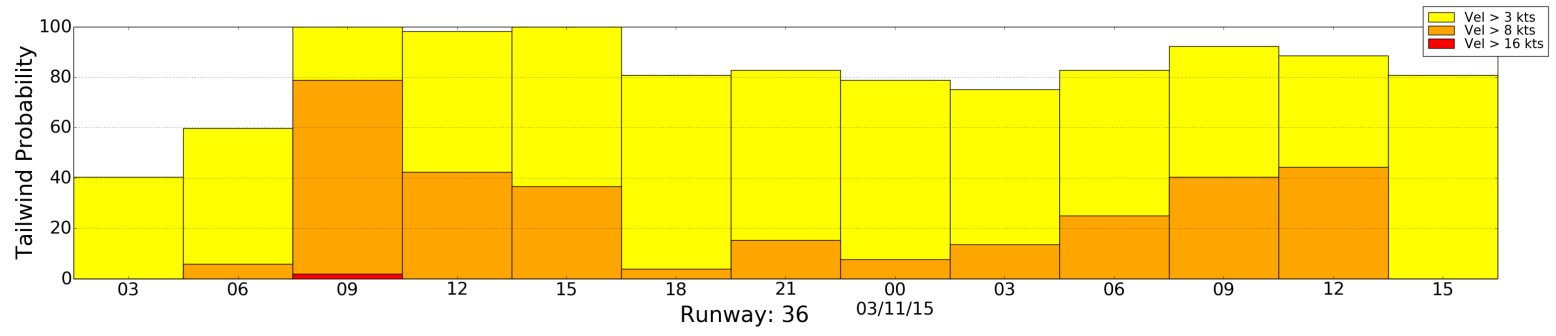

Figura 31.7: GLAMEPS: Viento en cola Cabecera 36 LEMD (diagrama de barras). 


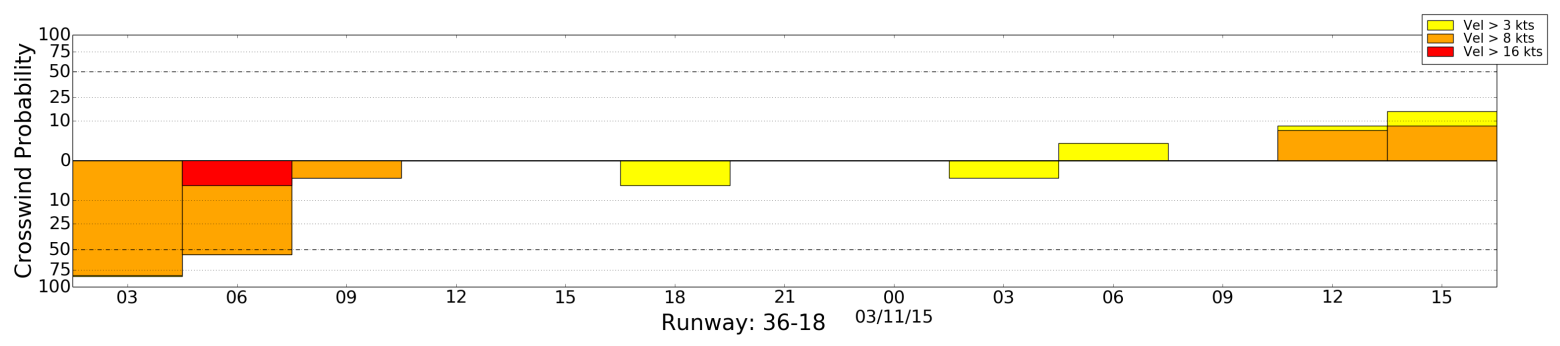

Figura 31.8: GLAMEPS: Viento cruzado Pista 36-18 LEMD (diagrama de barras).

\subsection{EPSgramas de alta resolución temporal y espacial: gamma- SREPS}

Nuestro siguiente paso es utilizar un SPC basado en modelos no hidrostáticos de alta resolución como el AEMET- $\gamma$ SREPS (cap. 22 en la página 333). Las salidas proporcionadas por este sistema tienen una resolución espacial de 2,5 km y temporal de 1 hora. Este nivel de resolución temporal permite utilizar EPSgramas continuos, donde las líneas se corresponden a los percentiles utilizados en el boxplot. Dado que en el momento de escribir este capítulo no se disponía todavía de datos del sistema $\gamma$ SREPS, en los gráficos mostrados se han utilizados los datos proporcionados por GLAMEPS para ejemplificar cómo resultarían los EPSgramas continuos.
Las Figuras 31.9 y 31.10 muestran, respectivamente, EPSgramas continuos para la velocidad del viento en LEMD y para la precipitación en LEBL.

El aumento de resolución temporal, que permite el paso de los boxplot a EPSgramas continuos, plantea un problema para la representación de la dirección del viento. Los diagramas de sectores de viento no permiten visualizar correctamente un número grande de pasos temporales, como puede verse en la Figura 31.11 en la página siguiente, parte izquierda. Para resolver este problema se optó por diseñar un diagrama matricial en el que la gama de colores se asocia a la probabilidad de cada sector de dirección del viento. El resultado sería el de la Figura 31.11 en la página siguiente, parte derecha.

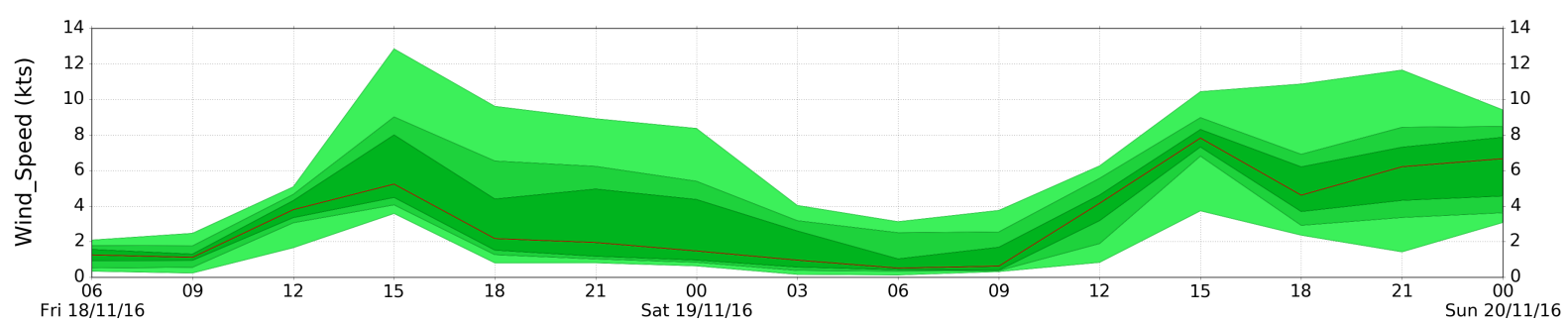

Figura 31.9: GLAMEPS: Velocidad Viento LEMD.

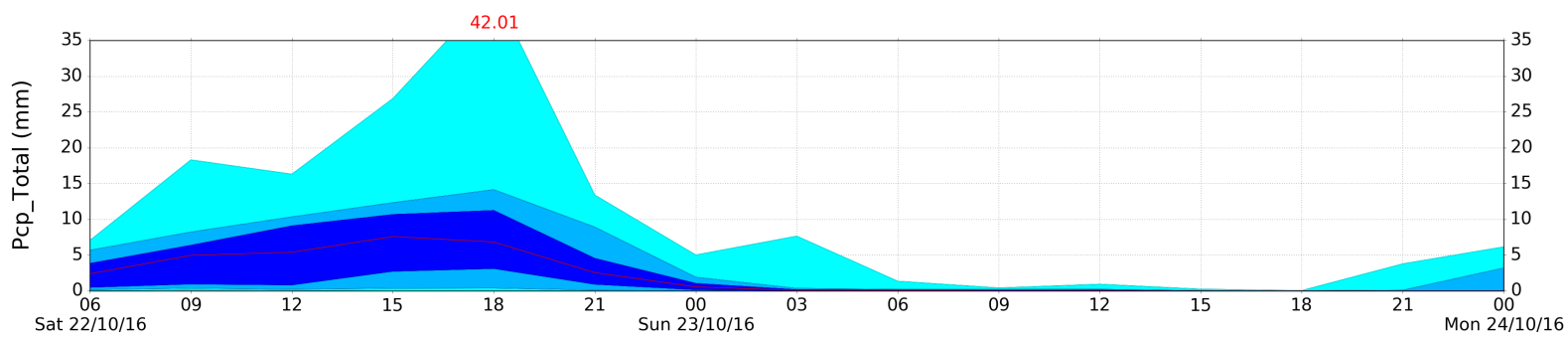

Figura 31.10: GLAMEPS: Precipitación LEBL. 

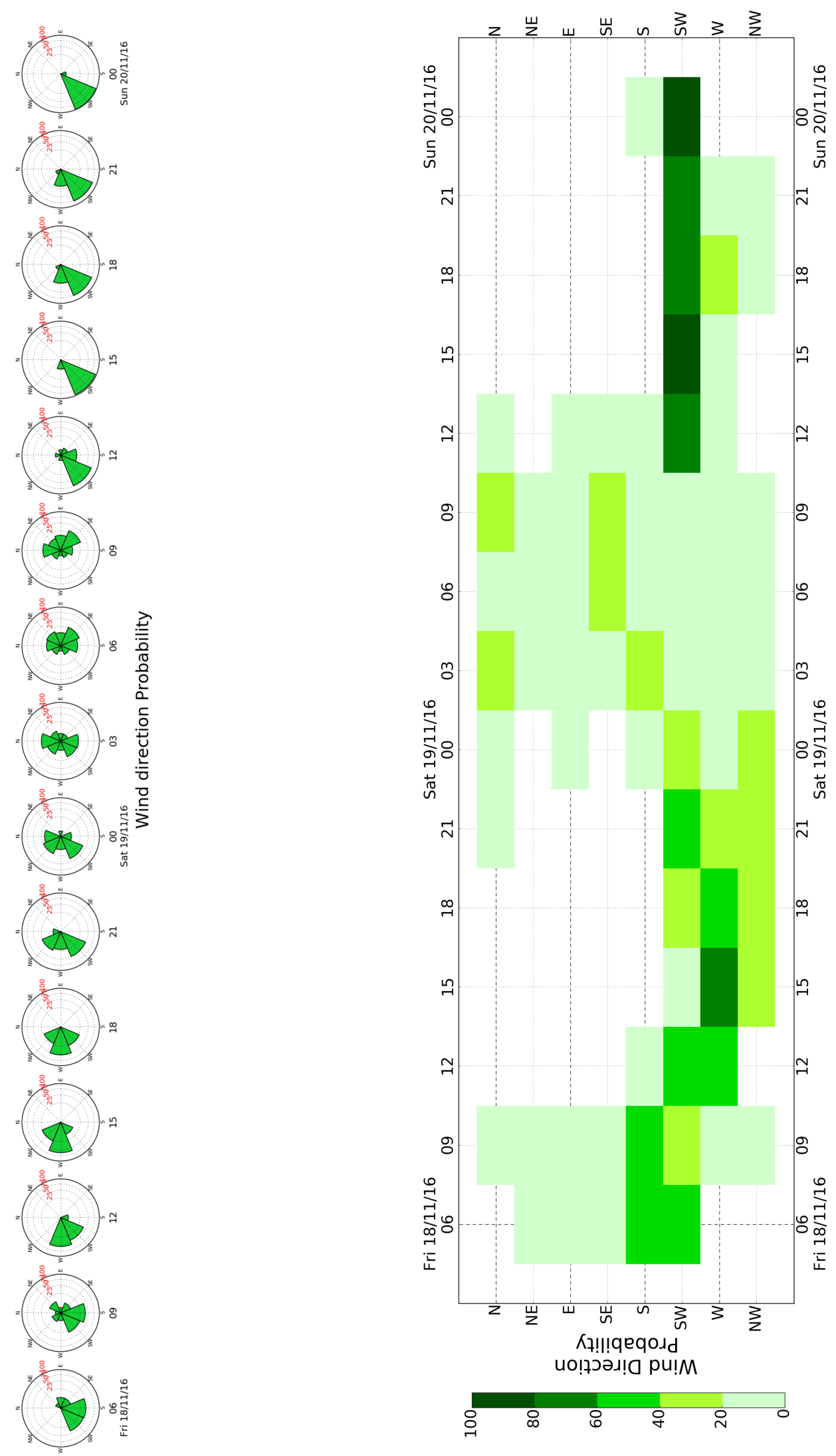

Figura 31.11: EPSgramas realiuzados basados en datos del SPC GLAMEPS. Izquierda: Velocidad del Viento LEMD (Sectores de Probabilidad). Derecha: Dirección del Viento (Diagrama Matricial). 
Con esta representación matricial para la dirección del viento se puede aumentar la resolución temporal sin que afecte a la compresión visual del EPSgrama. Esta solución permite visualizar hasta 48 horas la dirección del viento y su probabilidad en un gráfico muy compacto. Los EPSgramas aeronáuticos de diagrama de barras de Viento en cola en una cabecera y Viento cruzado en una pista se mantendrían sin cambios respecto a los realizados con el GLAMEPS. Cabe señalar que el aumento de resolución espacial permite asociar a un aeropuerto varios puntos de grid, con lo que se podrían elaborar EPSgramas más complejos. Y, por último, mencionar que el software está realizado con Python $[2,3]$ https: //www . python . org/ y su librería gráfica Matplotlib [1] https://matplotlib.org/.

\subsection{Anexos}

Nombres OACI. La Organización de Aviación Civil Internacional (OACI) es el organismo internacional encargado, entre otras cosas, de la generación de normativas y en materia de coordinación y seguridad en aviación civil (ICAO en inglés). Cada aeropuerto recibe un código OACI que se compone, por un lado, de dos letras iniciales: la primera letra indica la región, la segunda el país dentro de esa región. Por ejemplo: $L$ región sur de Europa, $E$ España (excepto Canarias, Ceuta y Melilla). El código OACI se compone, además, de dos letras que indican el aeropuerto. Los aeropuertos que aparecen en el texto de este capítulo son $L E B L$, Barcelona-El Prat y $L E M D$, Madrid-Barajas Adolfo Suárez. Los aeropuertos De Canarias o de Ceuta y Melilla, al estar en otra región OACI, comienzan con GC y GE, respectivamente.

TAF (Terminal Aerodrome Forecast). Es un pronóstico del tiempo meteorológico en un aeródromo que se realiza normalmente cada 6 horas y con validez de 24 horas. Ejemplo de TAF para Barcelona-El Prat:

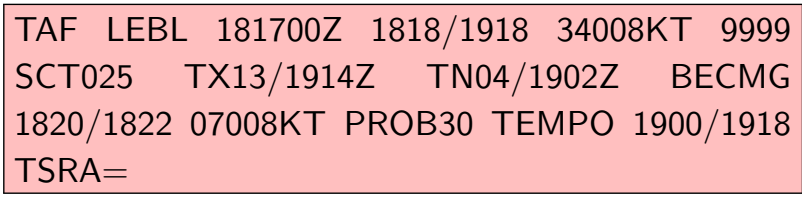

Está codificado y contiene un pronóstico de los siguientes parámetros: viento (34008KT), visibilidad (9999), nubosidad (SCT025), temperatura máxima y mínima (TX13/1914Z TN04/1902Z), tiempo presente (TSRA). Aparecen términos de evolución temporal (BECMG, TEMPO) y probabilistas (PROB30).

METAR (METeorological Aerodrome Report). Es una información del tiempo meteorológico presente en un aeródromo que se realiza normalmente cada media hora. Ejemplo de METAR para Madrid-Barajas Adolfo Suárez:

METAR LEMD 012200Z 23004KT 8000 -RA
BKN022 08/07 Q1014=

Está codificado y contiene información actual de los siguientes parámetros: viento (23004KT), visibilidad (8000), tiempo presente (-RA), nubosidad (BKN022), temperatura y punto de rocío $(08 / 07)$ y, finalmente, presión reducida al nivel del mar (Q1014). 


\subsection{Referencias}

[1] Hunter, J D. "Matplotlib: A 2D graphics environment". En: Computing In Science \& Engineering 9.3 (2007), páginas 90-95. DOI: 10.1109/MCSE . 2007.55 (citado en página 529).
[2] Rossum, G van. "Python tutorial, Technical Report CS-R9526”. En: (1995). URL: https : / / docs . python . org / 3 / tutorial/ (citado en página 529).

[3] Rossum, G van. "Python Language Reference, version 2.7". En: (2016). URL: https: / / docs . python . org / 2 / reference / index.html (citado en página 529). 
